\title{
Numerical Investigation of a Squeezing Flow Between Concentric Cylinders Under the Variable Magnetic Field of Intensity
}

Muhammad Sohail Khan ( $\sim$ sohailkhan8688@gmail.com )

Jiangsu University

Shabnam Shabnam

Jiangsu University

\section{Research Article}

Keywords: Squeezing flow, Heat Transfer, Variable Magnetic Field, Concentric Cylinders, Entropy Generation, Parametric Continuation Method (PCM) and BV4C Schemes

Posted Date: January 19th, 2022

DOl: https://doi.org/10.21203/rs.3.rs-1255296/v1

License: (c) (i) This work is licensed under a Creative Commons Attribution 4.0 International License.

Read Full License 


\title{
Numerical Investigation of a Squeezing Flow Between Concentric Cylinders Under the Variable Magnetic Field of Intensity
}

\author{
Shabnam ${ }^{(1)}$, Muhammad Sohail Khan ${ }^{(1) *}$ \\ shabnam8688@gmail.com, sohailkhan8688@gmail.com \\ (1) School of Mathematical Sciences, Jiangsu University, Zhenjiang 212013, Jiangsu, China
}

\section{Abstract}

The theme of the ongoing research is to examine the mass and heat transmission phenomena of squeezing flow between two concentric cylinders under the effect of heat sources and magnetic fields. The impacts of the Lorentz force on the behaviour of the liquid flow is elucidated via a magnetic field which is incorporated in the momentum equation. Moreover, the term $Q=\frac{Q_{0}}{1-\beta t}$ has been used as a source/sink within concentric cylinders. The proposed model of PDEs formulates the physical phenomena of time-dependent incompressible two-dimensional squeezing flow via modified Navier-Stokes equation, energy equation and mass transfer equation and variable magnetic field. The proposed model involved a highly nonlinear system of PDEs which has been reduced into a system of ODEs via Lie group of similarity transformation and subsequently solved numerically in MATLAB by Parametric Continuation Method (PCM). The direct impact of the squeezing parameter on the profile of temperature and concentration have been observed. Furthermore, an increment in the heat source indicates a decrement in the liquid temperature profile, while an inverse relationship is observed for the concentration profile. In addition, the declining effect of Soret number on the concentration profile is noticed. Beside this, a direct impact of $\operatorname{Pr}, \Omega$ and $E c$ on the entropy generation has been noticed, while an opposite impact has been noticed at Bejan number. The numerical outcomes of the proposed model that explain the characteristics of fluid flow are quantitatively elucidated by tables, and displayed graphically.

Keywords: Squeezing flow, Heat Transfer, Variable Magnetic Field, Concentric Cylinders, Entropy Generation, Parametric Continuation Method (PCM) and BV4C Schemes.

\footnotetext{
${ }^{*}$ Corresponding Author: sohailkhan8688@gmail.com
} 


\section{Introduction}

Work on the movement of liquids with stretching cylinders has attracted the attention of many researchers. It should be noted that the flow of the boundary layer due to surface shrinkage or stretching is a related type of flow that is reflected in several engineering and industrial processes. There are many uses of such flow in the engineering process, for instance in the polymer and metallurgy process such as extraction and manufacturing of polymer and rubber sheets, melting, hot rolling, paper manufacturing, wire drawing and glass fibre manufacturing.

It is noteworthy that the unsteady nature of many liquid movements plays a more practical role and has gained considerable attention in recent years. In several applications, the ideal liquid flow problems around the instrument is not usually steady, but the undesirable volatile effects are either due to the body's own involvement or due to fluctuations or inconsistencies in the surrounding fluid. Otherwise, some equipments require time-dependent movement of fluid to perform their basic functions has been analyzed by McCroskey [1]. The work on the time-dependent boundary layers is important because of the fact that all boundary layers, which exist in practice, are in a sense, time-dependent. Time-dependent viscous fluid movement has been extensively studied and all the features of transient impacts are now less or more familiar to the researcher in the field of mechanics. Wang [2] analyzed the incompressible steady viscous liquid movement outside the stretching hollow cylinder while the fluid was not flowing. The same problem has been expanded through incorporating the effects of suction and injection by Ishak et al. [3]. Telionis [4], Wang [5] and Riley [6] have summarized the basic ideas and significant contributions on the subject. A better interpretation of unsteady liquid flow and the application of this approach to newaly design methods provide significant enhancements in the performance, reliability and price of several fluid dynamic equipments.McLaughlin and Wang [7] seem to have been the first in the field to study the fluid flow at a shrinking surface. To adjust this fluid flow, the liquid is pushed in slot direction and the fluid flow is completely unlike from the stretching case. These authors have shown that massive suction is usually required to maintain flow on a shrinking sheet. Another instance of fluid moving towards a shrinking sheet is the incrementing shrinkage done by Wang, [8]. It has been reported that injection reduces the rate of heat transfer and skin friction to the surface whereas suction works in the opposite direction.

In recent past, Fang et al. [9] investigated the time-dependent viscous fluid movement on an extended stretching cylinder that gives the exact solution of the Navier-Stokes equation. They pointed out that due to the expansion of the cylinder there is a reverse flow and the flow field is firmly affected by the Reynolds number and unsteady parameter. Later on, Fang et al. [10] studied the time-dependent viscous fluid flow numerically in the outside region of the extending or contracting type cylinder. Ste- 
fan, [11] presented his own work on squeezing fluid flow under lubricating approximation approach. Domairry and Aziz, [12] have analyzed the impacts and properties of a squeezing flow of viscous liquid under the magnetic field between two parallel discs. Siddiqui et al. [13] investigated the impact of hydro-magnetic squeezing flow of the viscous liquid between two long parallel plates. In both of these studies, the models were solved using the Homotopy Perturbation Method (HPM). likewise, Rashdi et al. [14] studied the hydrodynamic of the viscous squeezing fluid flow using Homotopy Analysis Method (HAM).

The unsteady/steady electroosmotic liquid motion through an infinite expandand cylindrical channel of diameters 10 to $100 \mathrm{~nm}$ has been investigated by Nayak [15]. They have used a combination of $(\mathrm{NaCl}+\mathrm{H} 2 \mathrm{O})$ for numerical computation of mass, velocity, potential, and mixing efficiency. For the channel diameters the outcomes are acquired in both steady and unsteady cases for small, large or equal than electric double layer (EDL). Likewise, Shekholeslami et al. [16] analyzed the behaviour of time-dependent nanofluid flow between two parallel squeezing plates, and solved the proposed model by $(\mathrm{ADM})$, and concluded that the Nusselt number increments with the increment in the volume fraction and Eckert number, whereas it reduces with the enhancement of the squeezing number. Rajvanshi et al. [17] used the Brinkman model to investigate the MHD squeezing flow of viscous non-compressible liquid in a porous medium between two permeable revolving plates. Sangapatnam et al [18] studied the radiation and mass transfer impact on the MHD flow past of natural convection through iso-thermal vertical plate under viscous dissipation. The impact of radiation, MHD, and mass transfer on the transient flow of natural convection through hot vertical porous plates in the presence of viscous dissipation was analyzed by Prasad and Reddy [19]. Rajvanshi et al. [20] studied the incompressible viscous squeezing fluid flow between two highly permeable revolving plates in the porous medium. Solutions are acquired for relatively low density optically thin mediums, and are suitable for liquids with a thickness much higher than wall roughness.

Pattnaik et al [21] analyzed the coupled effects of convective heating, Ohmic electrothermal dissipation and exponential heat source on magnetized micropolar nanofluid on an expanded plate by shooting quadrature. The comprehensive impacts of conjugate wall conduction and convective boundary heating on the flow of magnetized thermo-solute layer from nonlinear long plate with hydrodynamic thermal concentration slip and radioactive heat transfer was analyzed by Uddin et al [22]. Hosseinzadeh et al. [23] was performed the hydrothermal assessment with conventional fluid of ethylene glycol-water (50\%-50\%) consisting hybrid nanomaterials (TiO2-MoS2) in octagon with elliptical cavity in the center. Their outcomes showed that with an increment in the Rayleigh number from 10 to 100, the mean Nusselt number improved by about $(61.82 \%)$. The flow of hybrid nanofluid 
obtained by the combination of ethylene glycol-water (50\%-50\%) with nanomaterials (MWCNT-Ag) at a vertical stretching cylinder was studied by Hosseinzadeh et al. [24]. The outcomes indicated that, the spherical ana lamina geometries of nanomaterials have larger variation, and lamina gives (6\%) smaller value. They also indicated that with rising Hartman numbers, the radial velocity for hybrid nanomaterials decreased by (9.68\%). Umavathi et al [25] analyzed the influence of magnetic nanomaterials in incrementing the transport of heat in a tribological system under connective type heating boundary conditions (Robin). Rehan et al. [26-28] studied the augmented viscosity pattren and heat transfer behaviour of a transient two-dimensional non-compresssible squeezing flow of ion-nano-fluid between two long parallel concentric cylinders. To study heat transfer capacity, three different kinds of nanocomponents like Titanium oxide, Copper and Aluminum of volume fraction from 0.1 to $0.7 \mathrm{~nm}$ are suspended into ionic fluid in turns. The Maxwell Garnet model of thermal conductivity and Brinkman model of viscosity for nanomaterials have been adopted.

Khilap et al. [29], studied the influence of slip velocity, magnetic field and concentration for an unsteady fluid flow and heat transfer on parallel plates. For its numerical results, they have used Range-Kutta of order (four-five) in the frame of shooting techniques which produced interesting results for the following parameters as Schmidt number, Hartman number, Nusselt number, velocity and concentration on Sherwood number, velocity slip parameter, Skin friction, squeeze number and volume fraction of nanoparticle on temperature. Further, Siddiqui et al. [30], studied the hydromagnetic influences of a viscous fluid in horizontal parallel plates and the proposed model is solved by HPM. Sheikholeslami et al. [31], investigated the effect of forced convection heat transfer and nano-uniform magnetic field on nanofluid and found interesting results and the proposed model was then solved by the numerical scheme. Further, Sheikholeslami et al. [32], studied the nanofluid flow in two-stage simulation with heat transfer on parallel plates, and the constructed model is solved by HPM. Next, Sheikholeslami and Ganji [33], examined the provision of mass transfer and heat transfer in a parallel channel on unsteady nanofluid in the viscous dissipation existence and the effect of radiation. A group Okango et al. [34], studied Hall current effect in the existence of variable magnetic fields in the vertical porous flat plates and the flow between them. The flow is steady and the selected domain is laminar, but the whole system rotates with a uniform angular velocity around the normal axis of the plate. Bejan [35, 36], suggested in their proposed model that the flow of variable parameters can be taken to reduce the irreversibility in the heat transfer process through specific convective. Hijleh et al. [37], observed through a rotating cylinder the laminar mixed convective of the entropy and concluded that the increase in buoyancy parameter and Reynolds number occur due to the increase of entropy generation. Tasnim et al. [38], reviewed the study of the first law and 
second law of thermodynamics regarding the flow properties and heat transfer in the presence of a magnetic field through two parallel vertical plates with a porous medium. Odat et al. [39], studied the effects of entropy generation through the laminar flow past flate plate in the existence of magnetic field. They noticed that the magnetic field intensity lead to increased the rate of entropy generation. We can readily review from the above-mentioned literature that Rehan et al. [26] examined the impact of a changeable magnetic field in a tribological process between two concentric cylinders regarding the enhancement of heat and mass transfer. The process that has been scrutinized, comprises the transmission of Newtonian magnetic lubricant between two concentric cylinders under the influence of magnetism. The present study is about the entropy investigation of two-dimensional fluid movement, and the heat transfer with the coupled Lorentz force in the presence of heat sink/source. The numerical outcomes of different emerging parameters like heat source $H s$, Schmidt number $S c$, Prandtl number $\operatorname{Pr}$, Magnetic parameter $M$, Bejan number $B e$, squeezing number $S$, magnetic field, skin friction coefficient, Nusselt number, and entropy production are computed and subsequently displayed through various tables and graphs. The proposed model of the fluid flow has several applications in the field of transportation, biomedical, industrial and electronics.

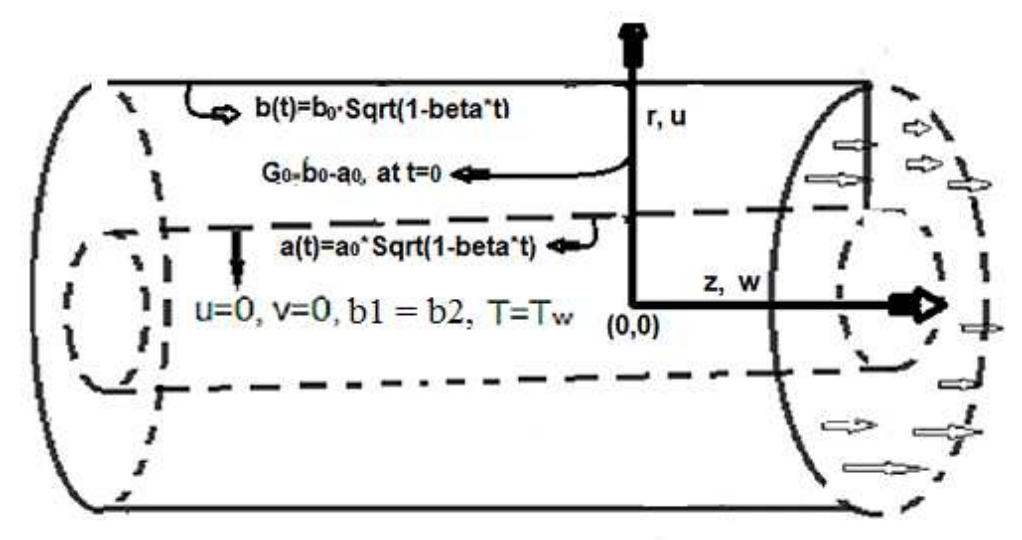

Figure 1: Geometry of the problem with Coordinate system

\section{Formulation}

We consider time-dependent two-dimensional laminar liquid boundary layer flow between two concentric cylinders in the presence of a variable magnetic field to analyze the behaviour of heat transfer. It is presumed that the liquid updated in the positive $z$-direction is infinite. The coordinate system in the polar form $(r, 0, z)$, has been taken at the centre of the inside cylinder, and hence the velocity components $u$ and $w$ lie along the direction of $r$-axis and $z$-axis respectively, as displayed in Figure 
(1). It is presumed that the diameters of the concentric cylinder dependent on time, and its timedependent radii $a(t)=\sqrt{1-\beta t}$ and $b(t)=\sqrt{1-\beta t}$ of the inside and outside cylinders respectively , where $b$ represents the extension/contraction power, $t$ shows time, and $a_{0}$ shows radius of the inside cylinder at $t=0$. The liquid is assumed to be symmetric flow with fixed viscosity $\mu$, and density $\rho$. Further, the heat source $Q=\frac{Q_{0}}{1-\beta t}$, is employed between the two concentric cylinders.

The mathematical formulation of the proposed model by continuity, momentum, magnetic field, energy and mass transfer equations are as follow [26].

Continuity equation:

$$
\frac{1}{r} \frac{\partial}{\partial r}(r u)+\frac{\partial w}{\partial z}=0
$$

The Momentum equations with variable magnetic effect [26-28]:

$$
\begin{gathered}
\frac{\partial u}{\partial t}+u \frac{\partial u}{\partial r}+w \frac{\partial u}{\partial z}=-\frac{1}{\rho} \frac{\partial P}{\partial r}+\frac{\mu}{\rho}\left(\frac{\partial^{2} u}{\partial r^{2}}+\frac{1}{r} \frac{\partial u}{\partial r}-\frac{u}{r^{2}}+\frac{\partial^{2} u}{\partial z^{2}}\right)-\frac{b_{3} \sigma}{\rho}\left(\frac{\partial b_{3}}{\partial r}-\frac{\partial b_{1}}{\partial z}\right) \\
\frac{\partial w}{\partial t}+u \frac{\partial w}{\partial r}+w \frac{\partial w}{\partial z}=-\frac{1}{\rho} \frac{\partial P}{\partial z}+\frac{\mu}{\rho}\left(\frac{\partial^{2} w}{\partial r^{2}}+\frac{1}{r} \frac{\partial w}{\partial r}+\frac{\partial^{2} w}{\partial z^{2}}\right)+\frac{b_{1} \sigma}{\rho}\left(\frac{\partial b_{3}}{\partial r}-\frac{\partial b_{1}}{\partial z}\right)
\end{gathered}
$$

Maxwell Equations [27, 28]:

$$
\begin{gathered}
\frac{\partial b_{1}}{\partial t}=u \frac{\partial b_{3}}{\partial z}+b_{3} \frac{\partial u}{\partial z}-w \frac{\partial b_{1}}{\partial z}-b_{1} \frac{\partial w}{\partial z}+\frac{1}{\sigma \mu_{e}}\left(\frac{\partial^{2} b_{1}}{\partial r^{2}}+\frac{1}{r} \frac{\partial b_{1}}{\partial r}-\frac{b_{1}}{r}+\frac{\partial^{2} b_{1}}{\partial z^{2}}\right) \\
\frac{\partial b_{3}}{\partial t}=-u \frac{\partial b_{3}}{\partial r}-b_{3} \frac{\partial u}{\partial r}+w \frac{\partial b_{1}}{\partial r}+b_{1} \frac{\partial w}{\partial r}+\frac{1}{\sigma \mu_{e}}\left(\frac{\partial^{2} b_{3}}{\partial r^{2}}+\frac{1}{r} \frac{\partial b_{3}}{\partial r}+\frac{\partial^{2} b_{3}}{\partial z^{2}}\right)
\end{gathered}
$$

The Energy Equation [26]:

$$
\begin{aligned}
\frac{\partial T}{\partial t}+u \frac{\partial T}{\partial r}+w \frac{\partial T}{\partial z} & =\frac{\kappa}{\left(\rho C_{p}\right)}\left(\frac{\partial^{2} T}{\partial r^{2}}+\frac{1}{r} \frac{\partial T}{\partial r}+\frac{\partial^{2} T}{\partial z^{2}}\right) \\
& +\frac{Q}{\left(\rho C_{p}\right)}\left(T-T_{0}\right)
\end{aligned}
$$

The Mass Transfer Equation [29]:

$$
\begin{aligned}
\frac{\partial C}{\partial t}+u \frac{\partial C}{\partial r}+w \frac{\partial C}{\partial z} & =D_{B}\left(\frac{\partial^{2} C}{\partial r^{2}}+\frac{1}{r} \frac{\partial C}{\partial r}+\frac{\partial^{2} C}{\partial z^{2}}\right) \\
& -k_{0}\left(C-C_{0}\right)+\frac{D_{T}}{\left(T_{m}\right.}\left(\frac{\partial^{2} T}{\partial r^{2}}+\frac{1}{r} \frac{\partial T}{\partial r}+\frac{\partial^{2} T}{\partial z^{2}}\right)
\end{aligned}
$$

where $b_{1}, b_{3}$ are the components of magnetic field, $u$ and $w$ are the component of velocity field, $\left(\rho C_{p}\right)$ is the heat capacity, $P$ is fluid pressure, $T$ is the temperature, $\rho$ is fluid density, $\sigma$ is electrical conductivity, $\mu$ is kinematic viscosity, $\kappa$ is the thermal conductivity. 


\section{Boundary conditions}

The boundary conditions of the proposed model as follow [26]:

$$
\begin{aligned}
& u=w=0, b_{1}=-\frac{2 M_{0} \nu}{a_{0} \sqrt{\xi} \sqrt{1-\beta t}}, b_{3}=\frac{4 M_{0} \nu z}{a_{0}^{2}(1-\beta t)}, T=T_{m}-T_{0}, C=C_{m}-C_{0} \quad \text { at } \quad r=a(t) \\
& u=-\frac{2 \nu}{a_{0} \sqrt{\xi} \sqrt{1-\beta t}}, w=0, b_{1}=b_{3}=0, T=C=0, \quad \text { at } \quad r=b(t)
\end{aligned}
$$

The following similarity transformations have been used for reducing a system of PDEs $(1-7)$ into a non-linear system of ODEs,

$$
\begin{aligned}
& u=-\frac{2 \nu}{a_{0} \sqrt{\xi} \sqrt{1-\beta t}} F(\xi), \quad w=\frac{4 z \nu}{a_{0}^{2}(1-\beta t)} F^{\prime}(\xi), \quad b_{1}=-\frac{2 M_{0} \nu}{a_{0} \sqrt{\xi} \sqrt{1-\beta t}} G^{\prime}(\xi), \\
& b_{3}=\frac{4 z M_{0} \nu}{a_{0}^{2}(1-\beta t)} G^{\prime}(\xi), \quad \xi=\frac{r^{2}}{a_{0}^{2}} \frac{1}{1-\beta t}, \quad \theta(\xi)=\frac{T-T_{0}}{T_{m}-T_{0}}, \quad \phi(\xi)=\frac{C-C_{0}}{C_{m}-C_{0}}
\end{aligned}
$$

Therefore, equation (1) of the model has satisfied automatically, and the reduced form of the remaining equations $(2-7)$ are as follow:

$$
\begin{gathered}
F^{\prime \prime \prime \prime}=\frac{1}{\xi}\left(S\left(2 F^{\prime \prime}+\xi F^{\prime \prime \prime}\right)+F^{\prime} F^{\prime \prime}-F F^{\prime \prime \prime}+\frac{R e_{m} M}{2 \xi}\left(S G G^{\prime}+2 \xi S G^{2}-2 F G^{2}+2 F F^{\prime} G^{\prime}\right)\right. \\
+\frac{R e_{m} M}{\xi}\left(S G G^{\prime}-\frac{1}{2}\left(F^{\prime} G^{2}-F G G^{\prime}\right)+F^{\prime \prime} G^{2}\right) \\
+\frac{R e_{m}^{2} M S}{2 \xi^{2}}(\xi-F)\left(S G^{2}+2 \xi S G G^{\prime}-2 F G G^{\prime}+2 F F^{\prime} G\right) \\
\left.+\frac{R e_{m} M}{2 \xi^{2}}\left(S G^{2}+2 \xi S G G^{\prime}-2 F G G^{\prime}+2 F F^{\prime} G\right)\right)-\frac{2}{\xi} F^{\prime \prime \prime}, \\
G^{\prime \prime}=\frac{R e_{m} M}{2 \xi}\left(S\left(G+2 \xi G^{\prime}\right)-2 F G^{\prime}+2 F F^{\prime}\right), \\
\theta^{\prime \prime}=-\frac{1}{2 \xi} \theta^{\prime}+\frac{P r}{\xi}(S \xi-F) \theta^{\prime}+\frac{H s}{\xi} \theta, \\
\phi^{\prime \prime}=-\frac{1}{2 \xi} \phi^{\prime}+\frac{S c}{\xi}\left(S \xi \phi^{\prime}-F \phi^{\prime}+C_{1} S \phi+\frac{S r S c}{2 \xi} \theta^{\prime}-\frac{S c S r P r}{\xi}(S \xi-F) \theta^{\prime}-\frac{S c S r H s}{\xi} \theta,\right.
\end{gathered}
$$

and the boundary conditions in the reduced form as follow,

$$
\begin{aligned}
& F(1)=0, \quad F^{\prime}(1)=0, \quad G(1)=1, \quad \theta(1)=1, \quad \phi(1)=1, \quad \text { at } \quad r=1 \\
& F(k)=1, \quad F^{\prime}(k)=0, \quad G(k)=0, \quad \theta(k)=0, \quad \phi(k)=0, \quad \text { at } \quad r=k
\end{aligned}
$$

where $M=\frac{M_{0}^{2} \sigma_{f}}{\rho}$ Magnetic parameter, $S=\frac{a_{0}^{2} \alpha}{4 \nu}$ squeeze parameter, $R e_{m}=\sigma \nu \mu_{e}$ Rynold's Magnetic parameter, $\operatorname{Pr}=\frac{\nu(\rho C p)}{\kappa}$ Prandtl number, $H s=\frac{Q_{0} a_{0}^{2}}{\kappa}$ heat source parameter, $S c=\frac{\nu}{D_{m}}$ Schmidt number, $S r=\frac{D_{l}\left(T_{m}-T_{0}\right)}{C_{m}-C_{0}}$ Soret number, $C_{1}=\frac{k_{0}(1-\beta t)}{\beta}$ Chemical reaction parameter, $\frac{1}{C p}\left(\frac{2 \nu}{r}\right)^{2}$ Eckert number and $\Omega=\frac{T_{m}-T_{0}}{T_{h}-T_{0}}$ is the temperature difference. 


\section{Entropy Generation}

The volumetric rate in the magnetic field can be taken as a part of the entropy generation [27, 28].

$$
\begin{aligned}
N_{g}= & \frac{\kappa}{T_{m}-T_{0}}\left(\left(\frac{\partial T}{\partial r}\right)^{2}+\left(\frac{\partial T}{\partial z}\right)^{2}\right)+\frac{\mu}{T_{m}-T_{0}}\left(2\left(\frac{\partial u}{\partial r}\right)^{2}+2\left(\frac{u}{r}\right)^{2}+2\left(\frac{\partial w}{\partial z}\right)^{2}+\left(\frac{\partial u}{\partial z}+\frac{\partial w}{\partial r}\right)^{2}\right) \\
& +\frac{\sigma}{T_{u}-T_{0}}\left(u^{2} b_{3}^{2}+w^{2} b_{1}^{2}-2 u w b_{1} b_{3}\right)
\end{aligned}
$$

the transformed form as follow as,

$$
\begin{aligned}
N s=\frac{N g}{N g_{0}} & =\eta^{2} \theta^{\prime 2}+4 \Omega E c \operatorname{Pr}\left(F^{2}+4 \eta^{2} F^{\prime 2}-2 \eta F F^{\prime}\right) \\
& +4 \Omega E c \operatorname{Pr} \delta\left(\eta^{4} F^{\prime \prime 2}+M \eta^{2}\left(F^{2} G^{\prime 2} F^{\prime 2} G^{2}-2 G F^{\prime} F G^{\prime}\right),\right.
\end{aligned}
$$

where $V$ is the velocity vector, $\left(T_{m}-T_{0}\right)$ is a reference temperature $\kappa$ is the thermal conductivity, $N s=\frac{N_{g}}{N_{g 0}}$, is the rate of entropy generation, $N_{g 0}=\frac{\kappa\left(T_{h}-T_{0}\right)}{\left(T_{m}-T_{0}\right)^{2} r^{2}}$ is the entropy generation rate behavior,

$\Omega=\frac{T_{u}}{T_{l}}$ is the dimensionless temperature difference. Hence, the dimensionless form of the entropy generation as follow.

$$
N s=N_{H}+N_{f}+N_{m f}
$$

Where $N_{f}, N_{H}$ and $N_{m f}$ are denoting the entropy generation due friction of the fluid, entropy generation due to heat transfer and local entropy generation due to magnetic field respectively. The viscosity and heat transfer effects are described in the Bejan number as follows.

$$
B e=\frac{N_{H}}{N s}=\frac{\eta^{2} \theta^{\prime 2}\left(1-R_{1} \theta\right)}{N s} .
$$

Emerging physical parameters in the reduced form of system are the Nusselt number and skin friction coefficient, and can be defined as,

$$
C_{f}=\frac{1}{\nu r R e}\left(\frac{\partial w}{\partial r}\right)_{r=b(t)}, \quad N_{u}=-\frac{r \kappa\left(\frac{\partial T}{\partial r}\right)_{r=b(t)}}{k\left(T_{m}-T_{0}\right)}
$$

In case of Eq.(19), we get

$$
\frac{R e^{2}}{8 z} C_{f}=f^{\prime \prime}(2), \quad-\theta^{\prime}(2)=\frac{N_{u}}{2}
$$

\section{Numerical Solution by PCM}

In this section, optimal choices of continuation parameters are made through the algorithm of PCM [27] for the solution of non-linear equations $(10-13)$ with boundary conditions in equation (14): 


\section{- First order of ODE}

To transform the equations $(10-13)$ into first order of ODE's, consider the following

$$
\begin{aligned}
& F=s_{1}, \quad F^{\prime}=s_{2}, \quad F^{\prime \prime}=s_{3}, \quad F^{\prime \prime \prime}=s_{4} \\
& G=s_{5}, \quad G^{\prime}=s_{6}, \quad \theta=s_{7}, \quad \theta^{\prime}=s_{8}, \quad \phi=s_{9}, \quad \phi^{\prime}=s_{10}
\end{aligned}
$$

putting these transformations in Eqs. $(10-13)$, which becomes

$$
\begin{aligned}
& s_{4}^{\prime}=\frac{1}{\xi}\left(S\left(2 s_{3}+\xi s_{4}\right)+s_{2} s_{3}-s_{1} s_{4}+\frac{R e_{m} M}{2 \xi}\left(S s_{5} s_{6}+2 \xi S s_{6}^{2}-2 s_{1} s_{6}^{2}+2 s_{1} s_{2} s_{6}\right)\right. \\
&+\frac{R e_{m} M}{\xi}\left(S s_{5} s_{6}-\frac{1}{2}\left(s_{2} s_{5}^{2}-s_{1} s_{5} s_{6}\right)+s_{3} s_{5}^{2}\right) \\
&+\frac{R e_{m}^{2} M S}{2 \xi^{2}}\left(\xi-s_{1}\right)\left(S s_{5}^{2}+2 \xi S s_{5} s_{6}-2 s_{1} s_{5} s_{6}+2 s_{1} s_{2} s_{5}\right) \\
&\left.+\frac{R e_{m} M}{2 \xi^{2}}\left(S s_{5}^{2}+2 \xi S s_{5} s_{6}-2 s_{1} s_{5} s_{6}+2 s_{1} s_{2} s_{5}\right)\right)-\frac{2}{\xi} s_{4} \\
& s_{6}^{\prime}= \frac{R e_{m} M}{2 \xi}\left(S\left(s_{5}+2 \xi s_{6}\right)-2 s_{1} s_{6}+2 s_{1} s_{2}\right) \\
& s_{8}^{\prime}=-\frac{1}{2 \xi} s_{8}+\frac{P r}{\xi}\left(S \xi-s_{1}\right) s_{8}+\frac{H s}{\xi} s_{7} \\
& s_{10}^{\prime}=-\frac{1}{2 \xi} \phi^{\prime}+\frac{S c}{\xi}\left(S \xi s_{10}-s_{1} s_{10}+C_{1} S s_{9}+\frac{S r S c}{2 \xi} s_{8}-\frac{S c S r P r}{\xi}\left(S \xi-s_{1}\right) s_{8}-\frac{S c S r H s}{\xi} s_{7}\right.
\end{aligned}
$$

and the boundary conditions becomes

$$
\begin{aligned}
& s_{1}(1)=0, \quad s_{2}(1)=0, \quad s_{5}(1)=1, \quad s_{7}(1)=1, \quad s_{9}(1)=1, \quad \text { at } \quad r=1 \\
& s_{1}(k)=1, \quad s_{2}(k)=0, \quad s_{5}(k)=0, \quad s_{7}(k)=0, \quad s_{9}(k)=0, \quad \text { at } \quad r=k
\end{aligned}
$$

\section{- Introducing of parameter $p$ and we obtained ODEs in a p-parameter group}

To get ODE's in a p-parameter group, let we know p-parameter in Eqs. $(22-25)$ and therefore,

$$
\begin{aligned}
s_{4}^{\prime} & =\frac{1}{\xi}\left(S\left(2 s_{3}+\xi s_{4}\right)+s_{2} s_{3}-s_{1}\left(s_{4}-1\right) q+\frac{R e_{m} M}{2 \xi}\left(S s_{5} s_{6}+2 \xi S s_{6}^{2}-2 s_{1} s_{6}^{2}+2 s_{1} s_{2} s_{6}\right)\right. \\
& +\frac{R e_{m} M}{\xi}\left(S s_{5} s_{6}-\frac{1}{2}\left(s_{2} s_{5}^{2}-s_{1} s_{5} s_{6}\right)+s_{3} s_{5}^{2}\right) \\
& +\frac{R e_{m}^{2} M S}{2 \xi^{2}}\left(\xi-s_{1}\right)\left(S s_{5}^{2}+2 \xi S s_{5} s_{6}-2 s_{1} s_{5} s_{6}+2 s_{1} s_{2} s_{5}\right) \\
& \left.+\frac{R e_{m} M}{2 \xi^{2}}\left(S s_{5}^{2}+2 \xi S s_{5} s_{6}-2 s_{1} s_{5} s_{6}+2 s_{1} s_{2} s_{5}\right)\right)-\frac{2}{\xi} s_{4} \\
s_{6}^{\prime} & =\frac{R e_{m} M}{2 \xi}\left(S\left(s_{5}+2 \xi s_{6}\right)-2 s_{1}\left(s_{6}-1\right) q+2 s_{1} s_{2}\right) \\
s_{8}^{\prime}=-\frac{1}{2 \xi} s_{8}+\frac{P r}{\xi}\left(S \xi-s_{1}\right)\left(s_{8}-1\right) q+\frac{H s}{\xi} s_{7} & \\
s_{10}^{\prime}= & -\frac{1}{2 \xi} \phi^{\prime}+\frac{S c}{\xi}\left(S \xi s_{10}-s_{1}\left(s_{10}-1\right) q+C_{1} S s_{9}+\frac{S r S c}{2 \xi} s_{8}-\frac{S c S r P r}{\xi}\left(S \xi-s_{1}\right) s_{8}-\frac{S c S r H s}{\xi} s_{7}\right.
\end{aligned}
$$


- Differentiation by $p$, reaches at the following system w.r.t the sensitivities to the parameter-p

Differentiating the Eqs. $(27-30)$ w.r.t by $p$

$$
d_{1}^{\prime}=h_{1} d_{1}+e_{1}
$$

where $h_{1}$ is the coefficient matrix, $e_{1}$ is the remainder and $d_{1}=\frac{d p_{i}}{d \tau}, 1 \leq i \leq 10$.

- Cauchy Problem

$$
d_{1}=y_{1}+a 1 v_{1}
$$

where $y_{1}, v_{1}$ are vector functions. By resolving the two Cauchy problems for every component. We are satisfied then automatically to ODE's

$$
e_{1}+h_{1}\left(a 1 v_{1}+y_{1}\right)=\left(a 1 v_{1}+y_{1}\right)^{\prime}
$$

and left the boundary conditions.

\section{- Using by Numerical Solution}

An absolute scheme has been used for the resolution of the problem

$$
\begin{gathered}
\frac{v_{1}^{i+1}-v_{1}^{i}}{\triangle \eta}=h_{1} v_{1}^{i+1} \\
\frac{y^{i+1}-y^{i}}{\triangle \eta}=h_{1} y^{i+1}+e_{1}
\end{gathered}
$$

\section{- Taking of the corresponding coefficients}

As given boundaries are usually applied for $p_{i}$, where $1 \leq i \leq 10$, for the solution of ODE's, we required to apply $d_{2}=0$, which seems to be in matrix form as

$$
l_{1} \cdot d_{1}=0 \text { or } l_{1} \cdot\left(a 1 v_{1}+y_{1}\right)=0
$$

where $a 1=\frac{-l_{1} \cdot y_{1}}{l_{1} \cdot v_{1}}$

\section{$6 \quad$ Results and Discussions}

We have analyzed time-dependent non-compressible two-dimensional squeezing flow between concentric cylinders with respect to heat transfer, and behavior of the magnetic effect. The impacts of various emerging parameters, including Prandtl number $(P r)$,Magnetic number $(M)$, squeezing parameter $(S)$, Schmidt parameter $S c$, magnetic Reynolds number $\left(R e_{m}\right)$, Soret parameter $S r$, Chemical reaction parameter $C 1$, Eckert number $E c$ and difference temperature $\Omega$ have been investigated regarding 
Table 1: Comparison of the numerical results by two methods PCM and BVP4C for Skin friction and Nusselt number, with various physical parameters.

\begin{tabular}{|l|c|c|c|c|}
\hline & PCM & BVP4C & PCM & BVP4C \\
\hline$S$ & $f^{\prime \prime}(1)$ & $f^{\prime \prime}(1)$ & $-\theta^{\prime}(1)$ & $-\theta^{\prime}(1)$ \\
\hline 0.0 & -4.5583 & -4.5583 & 0.4785 & 0.4785 \\
0.2 & -4.7652 & -4.7652 & 0.6137 & 0.6137 \\
0.4 & -4.9765 & -4.9765 & 0.7754 & 0.7754 \\
0.6 & -5.1924 & -5.1924 & 0.9650 & 0.9650 \\
0.8 & -5.4129 & -5.4129 & 1.1829 & 1.1829 \\
1.0 & -5.6376 & -5.6376 & 1.4286 & 1.4286 \\
1.2 & -5.8666 & -5.8666 & 1.7007 & 1.7007 \\
1.4 & -6.0997 & -6.0997 & 1.9973 & 1.9973 \\
1.6 & -6.3367 & -6.3367 & 2.3158 & 2.3132 \\
\hline
\end{tabular}

Table 2: Comparison of the numerical results by two methods PCM and BVP4C for Skin friction and Nusselt number, with various physical parameters.

\begin{tabular}{|c|r|r|r|r|}
\hline & PCM & BVP4C & PCM & BVP4C \\
\hline$H a$ & $f^{\prime \prime}(1)$ & $f^{\prime \prime}(1)$ & $-\theta^{\prime}(1)$ & $-\theta^{\prime}(1)$ \\
\hline 0.0 & -6.0586 & -6.0586 & 2.3946 & 2.3946 \\
0.2 & -6.0811 & -6.0811 & 2.3952 & 2.3952 \\
0.3 & -6.0924 & -6.0924 & 2.3955 & 2.3955 \\
0.6 & -6.1263 & -6.1263 & 2.3963 & 2.3963 \\
0.8 & -6.1490 & -6.1490 & 2.3968 & 2.3968 \\
0.9 & -6.1604 & -6.1604 & 2.3971 & 2.3971 \\
1.2 & -6.1947 & -6.1947 & 2.3980 & 2.3980 \\
1.5 & -6.2292 & -6.2292 & 2.3988 & 2.3988 \\
1.8 & -6.2639 & -6.2639 & 2.3997 & 2.3997 \\
\hline
\end{tabular}


Table 3: Comparison of the numerical results by two methods PCM and BVP4C for Skin friction and Nusselt number, with various physical parameters.

\begin{tabular}{|c|r|r|c|c|}
\hline & PCM & BVP4C & PCM & BVP4C \\
\hline$P r$ & $f^{\prime \prime}(1)$ & $f^{\prime \prime}(1)$ & $-\theta^{\prime}(1)$ & $-\theta^{\prime}(1)$ \\
\hline 0.1 & -5.0839 & -5.0839 & 0.7529 & 0.7529 \\
0.5 & -5.0839 & -5.0839 & 0.7744 & 0.7744 \\
1.0 & -5.0839 & -5.0839 & 0.8013 & 0.8013 \\
1.5 & -5.0839 & -5.0839 & 0.8285 & 0.8285 \\
2.0 & -5.0839 & -5.0839 & 0.8557 & 0.8557 \\
2.5 & -5.0839 & -5.0839 & 0.8831 & 0.8831 \\
3.0 & -5.0839 & -5.0839 & 0.9106 & 0.9106 \\
3.5 & -5.0839 & -5.0839 & 0.9381 & 0.9381 \\
4.0 & -5.0839 & -5.0839 & 0.9657 & 0.9657 \\
\hline
\end{tabular}

the fluid flow, heat transmission, mass transfer, entropy generation and Bejan number in Figs. (214). It is noteworthy that the outcomes of our proposed model produce better outcomes than the current model in the literature. Tables (1-3) are described the numerical results of two important flow parameters, skin friction and Nusselt number, which are acquired in MATLAB by two different numerical schemes (BVP4C and PCM).

It is indispensable to record that the squeezing number $S$ explains the flow in the concentric cylinder as shown in Fig. 2(a), where $S>0$ represent the two cylinder moving apart and $S<0$ represent the opposite trend of the cylinder for the so-called squeezing flow. In this ongoing analysis the positive values of $(S>0)$ are taken. As long as the value of the squeezing number $S$ is incremeting, the horizontal velocity components decrements gradually. It is also necessary to mention that the influence of the magnetic field is declining the intensity of the velocity throughout the domain under consideration which gives existence to a new force is known as Lorentz force. This force acts in the opposite direction of the flow, when the magnetic field is employed in the orthogonal direction to the flow. Such kind of opposing force gets slow the velocity of the fluid. Figure 2(b) illustrates the impact of squeezing number $S$ at the horizontal components of the fluid velocity $f(\eta)$. Therefore, it is also noteworthy that incrementing the negative value of the squeezing number $S$ expands the flow channel, and thus, decrements the horizontal components of the velocity $f(\eta)$ with the increase in the absolute value of $S<0$. Figure 3(a) and 3(b) describe the behaviour of the squeezing parame- 
ter $S$ with respect to two parallel concentric cylinders, the channel of the flow between two parallel concentric cylinders expands, when $(S>0)$, as a result, the vertical components of the velocity $f^{\prime}(\eta)$ decrements in the domain $\eta<1.5$, and increments in the domain $\eta>1.5$. Similarly, the channel of the flow between two parallel concentric cylinders contracts, when $(S<0)$, as a result, the vertical components of the velocity $f^{\prime}(\eta)$ increments in the domain $\eta<1.45$, and decrements onwards. Figure 4 illustrates the impact of magnetic parameter $M$, while Figure. 5(a) explains the effect of magnetic Reynold's number $R e_{m}$ with respect to horizontal velocity in the flow direction. It can be seen in Figures (4) and 5(a) that the horizontal components of fluid velocity decrements with the increase in the Hatmman number $H a$ and magnetic Reynold's number $R e_{m}$. A transverse magnetic field, when it is employed orthogonally to the flow direction, which produces an opposing force is known as Lorentz force. This force acts opposite to the flow direction and hence, the velocity gets decrease but it is completely showing opposite behaviour for the flow domain, where $\eta>1.4$. The velocity profile is maximum in the centre of the annulus cylinder and the velocity profile follow a parabolic type shape vertically in the upward direction. Figure 5(a) illustrates the effect of the radius of the inner cylinder and the magnetic Reynolds number $R e_{m}$ on the magnetic profile which shows that the electron is moving from outer to inner, in a conventional sense, we can say the current is flowing from inner to outer. This is obvious that the profile of the magnetic field decrements with incrementing $R e_{m}$. Figure 6(a) illustrates that, as long as the value of the squeezing parameter $S$ increments, the profile of the magnetic field decrements due to existence of Lorentz force which gets slow the motion of the fluid. In addition, when $S<0$ the annulus formed by an outer and inner cylinder contracts and two the cylinders gets close to one another, this condition together with declining Lorentz force produces an unfavourable pressure gradient. Hence, the magnetic profile increments with the increase in the intensity of the squeezing parameter $S$, as shown in Fig. 6(b). It has been noticed from the graph, that the magnetic field can be utilized to improve the fluid flow when the plates are getting close, as a result, the squeezing number increments which augments the velocity profile in both cases. Figure $7($ a) explains the impact of $S$ on $\theta(\eta)$ profile. For higher value of $S$, the temperature profile rises, as the kinetic energy of the molecules rises and the attraction between molecules weakens. Therefore, the viscosity of a fluid decrements with incrementing temperature profile. Figure $7(\mathrm{~b})$ shows the increase in the attractive binding energy and hence, the temperature profile reduces. In Figure 8(a), it is displayed that, the concentration profile decrements with the augmenting value of the squeezing number $S>0$, and thus, more squeezing generates cooling effects in the viscous liquid system. Figure 8(b) depicts the impact of squeezing number $S$ on the concentration profile, it shows that the concentration profile augments with the rise in the squeezing number $S$ when $\eta<1.5$. Nevertheless, it 
decrements with the increments of these parameters when $\eta>1.5$. The increment in Prandtl number $\operatorname{Pr}$ increments heat transfer and and decrements mass transfer as shown in Fig. (9), and increments the kinetic energy of ions which move faster i.e. they move faster ie they move their bearing charge faster and as a result the conductivity increases. They are running their bearing charge fast and as a result the conductivity rises, and hence the temperature profile augments with augmenting Prandtl number, and the electrolytic conductors conductivity also rises. The ratio of momentum diffusivity to thermal diffusivity is referred to Prandtl number. Therefore, an increment in the Prandtl number indicates that the momentum spreads rapidly and that the velocity boundary layer is relatively thicker than the layer of the thermal boundary, resulting in less heat transfer through bulk motion. This means that the conduction is dominated by the convection. Therefore, the mass transfer decrements with an increment in $\mathrm{Pr}$. Impact of heat source $H s$ on concentration and temperature profiles are shown in Fig. (10). The temperature profile decrements and the mass transfer increments and at the same time becomes linear at the augmenting value of the heat source $H s$. Figure (11) explains the impact of the chemical reaction parameter $C 1$ at the concentration profile. We can observe from the graph that the concentration profile are greatly influenced by the values of the chemical reaction parameter, and hence retards in the flow domain. However, the behavior of the flow becoming opposite in the region where $\eta>1.6$. Figure 12(a) illustrates the impact of Schmidt number $S c$, it has been noticed that the increment in Schmidt number $S c$ decrements the mass transfer. It is also so clear from the figure that an increment in $S c$ causes a decrement in the thickness of the concentration boundary layer. The reality is that the larger values of $S c$, has smaller diffusivity of mass which causes thinner concentration boundary layer. Figure 12(b) explains the impact of Soret parameter $S r$ at the concentration profile. In this paper the following values of $S r$ are taken as $S r=0.1,0.4$, $0.7,1.0$, it shows that the values of Soret parameter are augmented. It can be seen from the figure that the concentration profiles reduces and become parabolic with the above given values of $S r$. A ratio of temperature variation to concentration variation is referred to Soret number, by definition of Soret number, diffusive species with larger Soret values reduces the concentration profiles.

Figures 13(a-c) illustrates behavior of entropy generation, it has been observed that entropy generation augments in the entire domain with the increment in $E c, \operatorname{Pr}$ and $\Omega$. Moreover, an increment is noticed in both $M$ and $\Omega$ close to the cylinder wall whereas minor fluctuation is seen in the middle of the cylinder. The impacts of entropy generation with the rising value of the $\operatorname{Pr}$ is sketched in Fig. 13(a). It has been observed that entropy generation is optimized for a fixed value at the stretching surface inside the domain and slows down as the distance from the sheet increases. Further, the surface of the stretching sheet is a good source of entropy generation because heat transfer and fluid 
friction are significant at the surface. The Ec is sketched in Fig. 13(b) described the thermal energy heating measurement, it shows that the Eckert number is producing more entropy under the influence of stretching surface. Since the sheet and its vicinity rises the viscous heating due to a rise in the $E c$ and temperature difference $\Omega$ in Fig. 13(c), and consequently, increase the molecular disorganization and therefore, Increases the entropy production within the boundary layer. Moreover, heat is a disorganized shape of energy that increases due to the increment in $E c$ and $\Omega$ of viscous heat, so entropy construction increases. Therefore, the construction of entropy will be minimal due to low viscous heating. No considerable impact of the magnetic parameter $M$ at the dimensionless form of total entropy generation is noticed in the middle of the channel as shown in Fig. 13(d). There is a consistency in the various curves of the entropy generation. That is why entropy generation in transient form is basically a relatively important magnetic random and magnetic parameter. The variation in Bejan number due to the fluctuation of $E c, M, \operatorname{Pr}$ and $\Omega$ is sketched in Figures 14(a-d). We have seen that graphs gradually becomes linear from parabolic, and reveal that the Bejan number inside the cylinder decrements with the increment in $P r$ and $E c$ in Fig. 14(a-b). In the middle region of the cylinder for $\eta=1.6$, the irreversible phenomenon has been observed in the Bejan number. The Bejan number in the flow system decrements with the rising value of $\Omega$ as shown in Figure 14(c). It has been observed that heat transfer dominates the irreversible flow process. The magnetic parameter $M$ is sketched in Fig. 14(d). Since $B e$ and $M$ are positively correlated at the upper permeable wall, while $B e$ decline at the lower permeable wall. No influence is observed at the point $\eta=1.58$ on $B e$. Irreversibility is dominant, Irreversibility is due to fluid friction on the lower permeable wall and due to heat transfer to the upper cylinder but due to these two factors, Irreversibility equally contributes at $\eta=1.58$ due to these two factors.
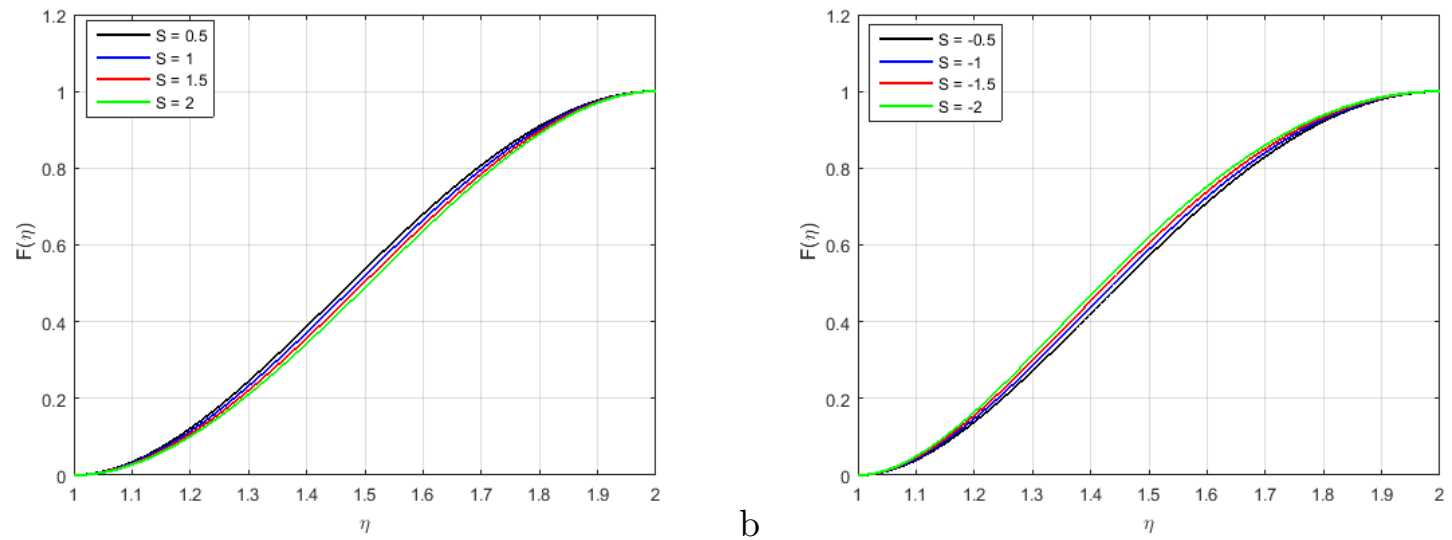

Figure 2: Influence of $F(\eta)$ for $S>0, S<0$ and fixed values of $\operatorname{Re}_{m}=2.5, M=0.5, \operatorname{Pr}=3.2, H s=$ $0.2, C 1=0.4, S c=0.4, S r=0.5$. 

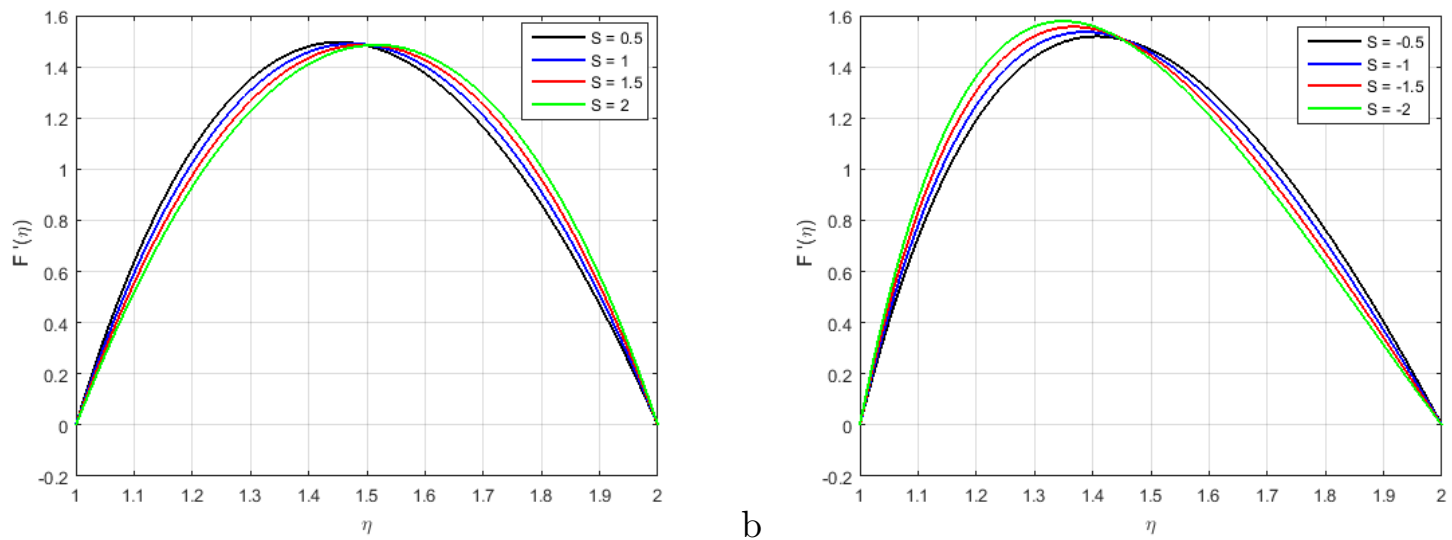

Figure 3: Influence of $F^{\prime}(\eta)$ for $S>0, S<0$ and fixed values of $R e_{m}=2.5, M=0.5, \operatorname{Pr}=3.2, H s=$ $0.2, C 1=0.4, S c=0.4, S r=0.5$.

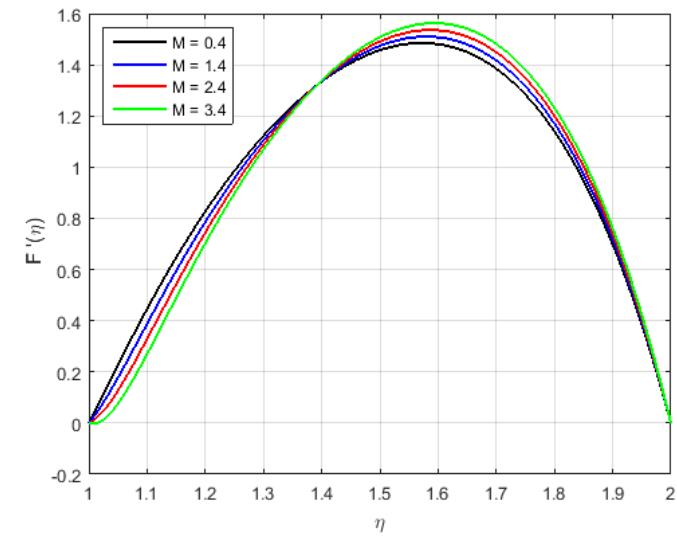

Figure 4: Influence of $F^{\prime}(\eta)$ for $M$ and fixed values of $S=2.5, \operatorname{Pr}=6.2, E c=0.5, \delta=0.4, \Phi_{1}=$ $0.02, \Phi_{2}=0.5, m=0.3$

\section{Concluding Remarks}

In the ongoing paper, we have studied the fluid flow, Maxwell equation, and mass and heat characteristics between two concentric parallel cylinders under the variable magnetic field to see the flow behaviour with respect to the following emerging parameters like a magnetic parameter, squeezing parameter, Soret number, Prandtl number, magnetic Reynold's number and heat sink/source. The proposed model which has been formulated the fluid flow by continuity equation, momentum equation, Maxwell equation and coupled energy and species equation with boundary conditions have been reduced into a set of highly nonlinear systems of ODEs by Lie group of similarity transformation. The proposed model is solved numerically by a highly efficient and extensively validated Parametric Continuation Method (PCM). In addition, the model outcomes and numerical scheme have been 

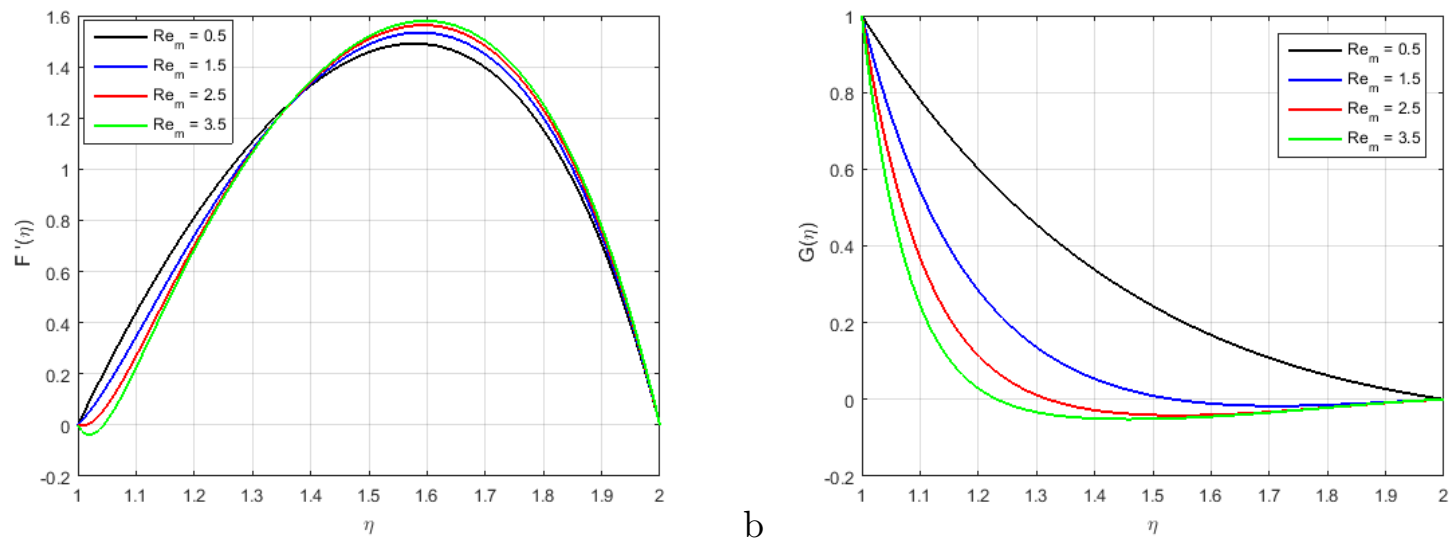

Figure 5: Influence of $F^{\prime}(\eta)$ and $G(\eta)$ for $R e_{m}$ and fixed values of $S=3.5, M=3.4, \operatorname{Pr}=6.2, H s=$ $1.2, C 1=1.4, S c=1.4, S r=1.5$.
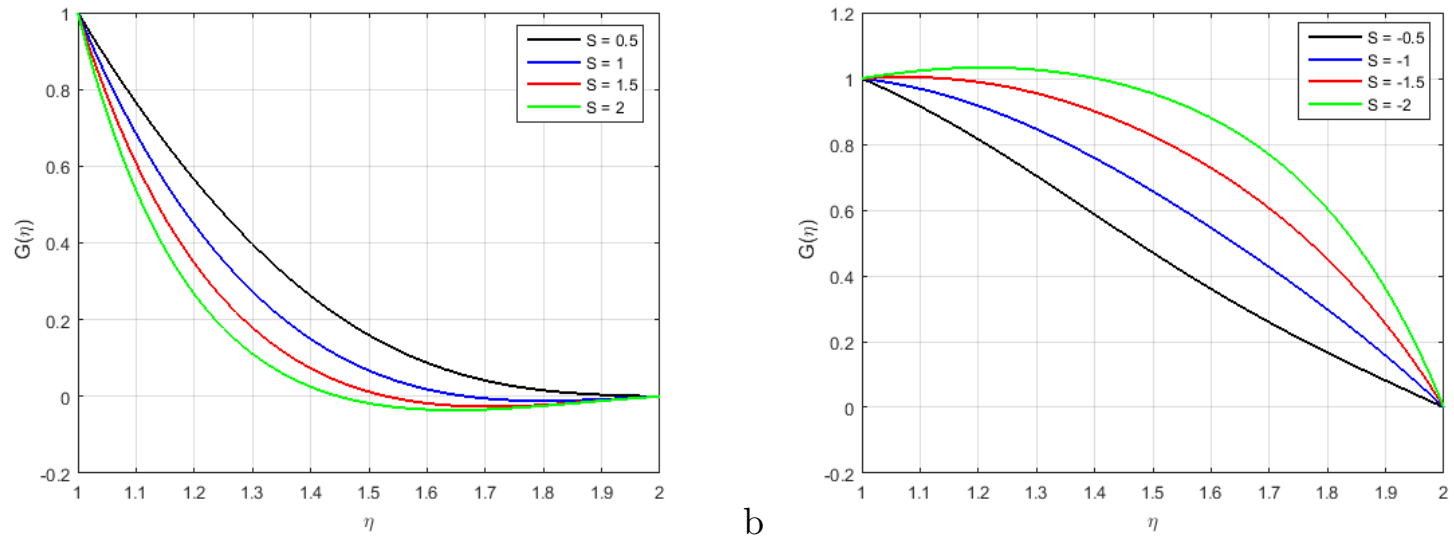

Figure 6: Influence of $G(\eta)$ for $S>0, S<0$ and fixed values of $\operatorname{Re}_{m}=2.5, M=0.5, \operatorname{Pr}=3.2, H s=$ $1.2, C 1=0.4, S c=1.4, S r=1.5$.

validated by solving the proposed model through another numerical scheme (BVP4C) in MATLAB and found a close correspondence. From the outcomes of the model, we have drawn the following conclusion:

- The squeezing number $S$ effect on the velocity profile reveals that the trend of the velocity profile is opposite and becomes parabolic.

- The temperature profile augments with augmenting values ??of the Prandle number.

- The contradicting behaviour between temperature and concentration profiles has been observed due to the augmenting values of the heat source parameter (Hs).

- The impact of Schmidt and Soret number at the concentration profile reveals that the incre- 

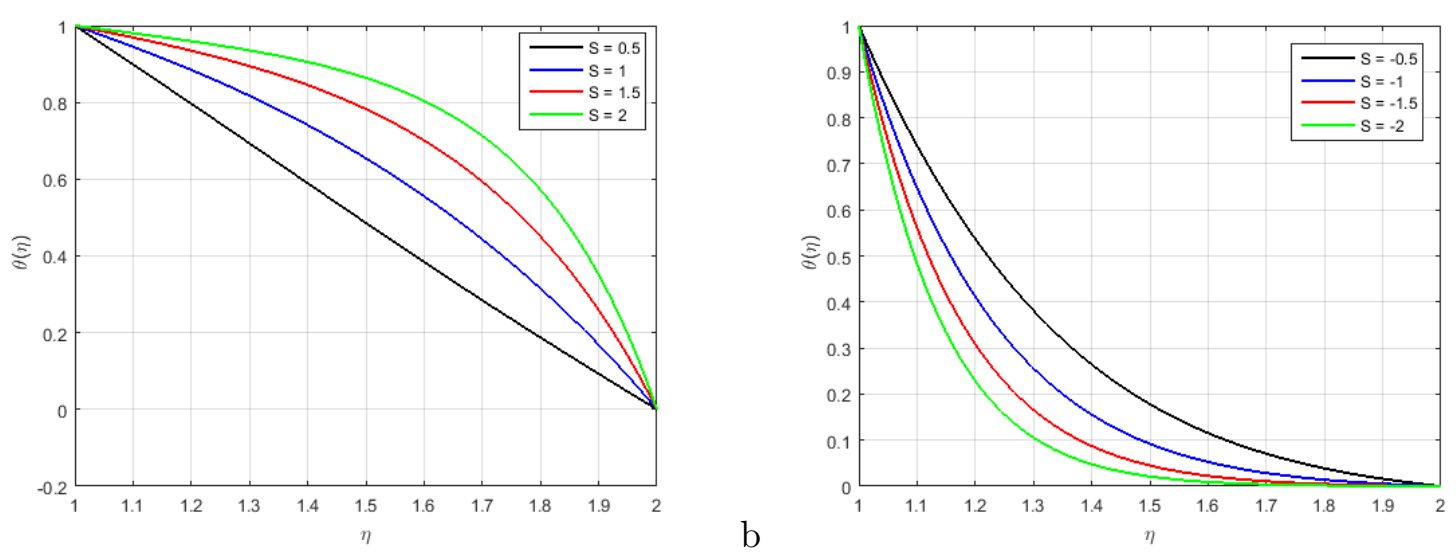

Figure 7: Influence of $\theta(\eta)$ for $S>0, S<0$ and fixed values of $R e_{m}=2.5, M=0.5, \operatorname{Pr}=3.2, H s=$ $1.2, C 1=0.4, S c=1.4, S r=1.5$.

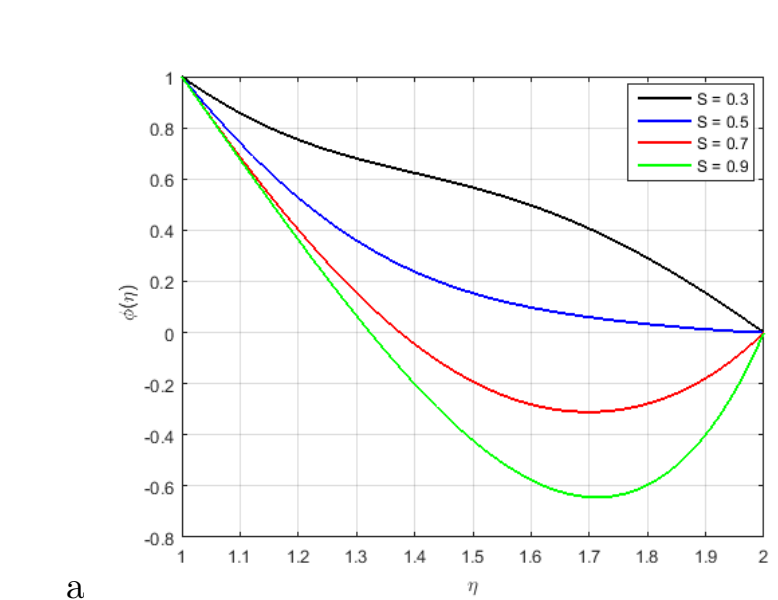

a $\mathrm{b}$

$\mathrm{b}$

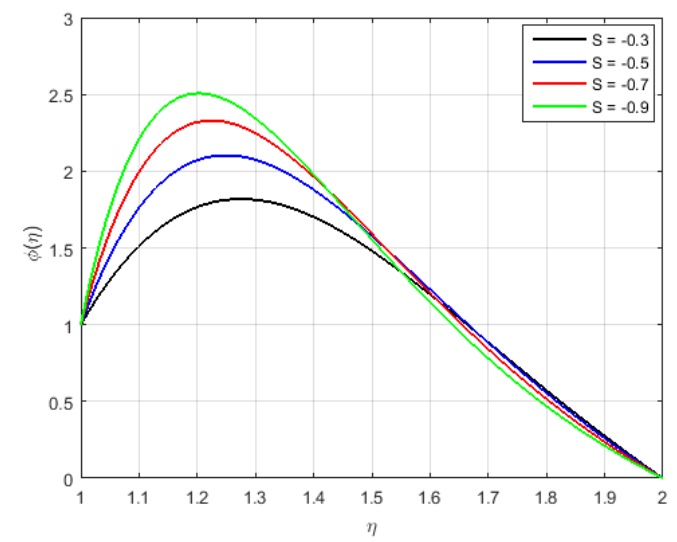

Figure 8: Influence of $\phi(\eta)$ for $S>0, S<0$ and fixed values of $R e_{m}=2.5, M=0.5, \operatorname{Pr}=6.2, H s=$ $1.2, C 1=1.4, S c=1.4, S r=2.5$.

menting values of Schmidt and Soret number decrements the concentration profile.

- The magnetic profile is reduced by the existence of the magnetic field due to the Lorentz force which slows down the movement of the fluid.

\section{Acknowledgments}

We acknowledge the insightful comments of editorial board to make this work more beautiful. 

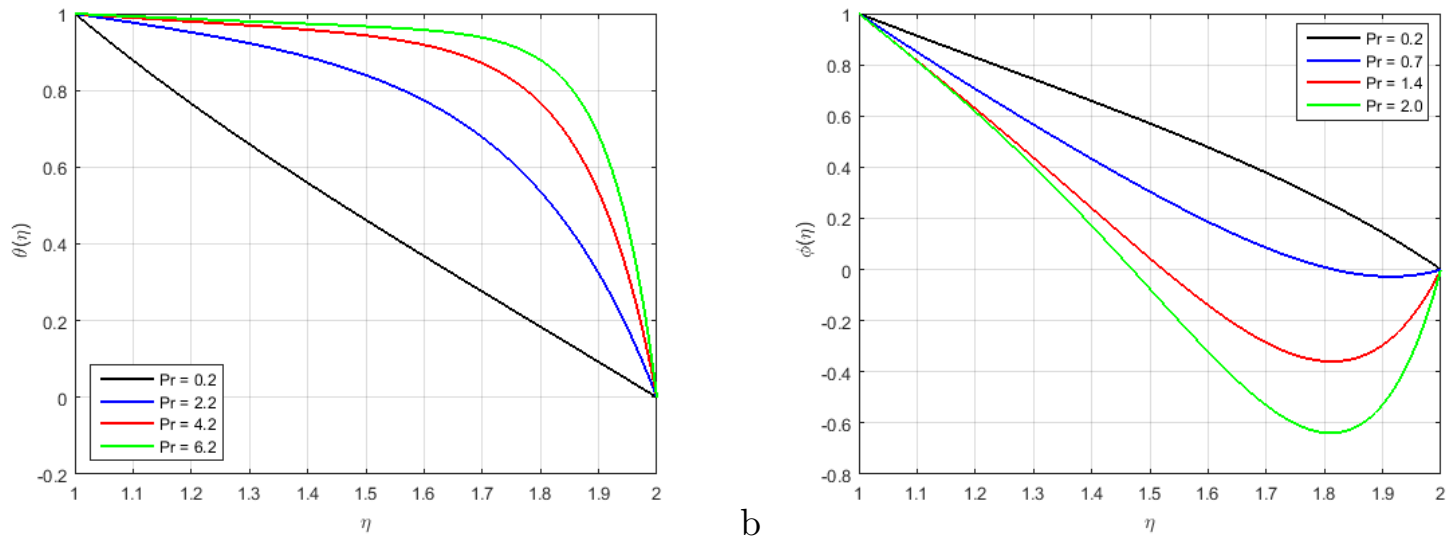

Figure 9: Influence of $\theta(\eta)$ and $\phi(\eta)$ for $\operatorname{Pr}$ and fixed values of $S=2.5, M=1.4, R e_{m}=2.5, H s=$ $1.2, C 1=1.4, S c=1.4, S r=2.5$.

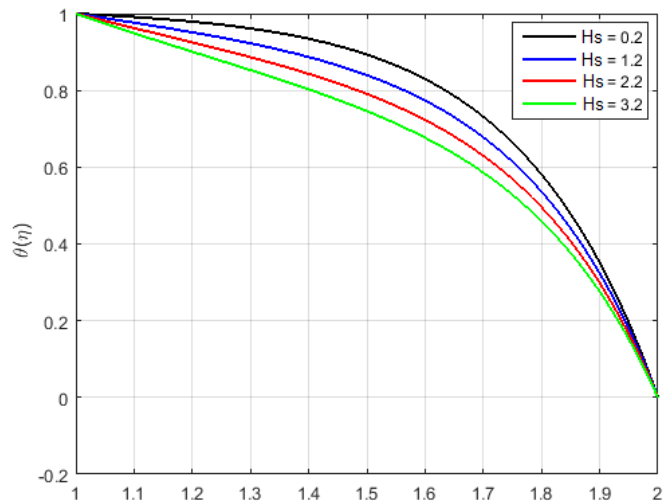

a

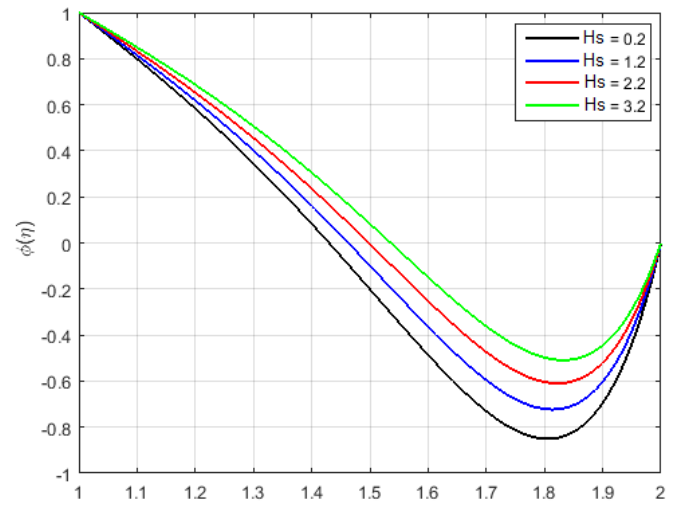

Figure 10: Influence of $\theta(\eta)$ and $\phi(\eta)$ for $H s$ and fixed values of $S=2.5, M=1.4, \operatorname{Pr}=2.2, \operatorname{Re}_{m}=$ $2.5, C 1=1.4, S c=1.4, S r=2.5$.

\section{Authors' contributions}

All authors contributed equally and significantly in writing this article. All authors read and approved the final manuscript.

\section{Institutional Review Board Statement}

Not applicable.

\section{Informed Consent Statement}

Not applicable. 


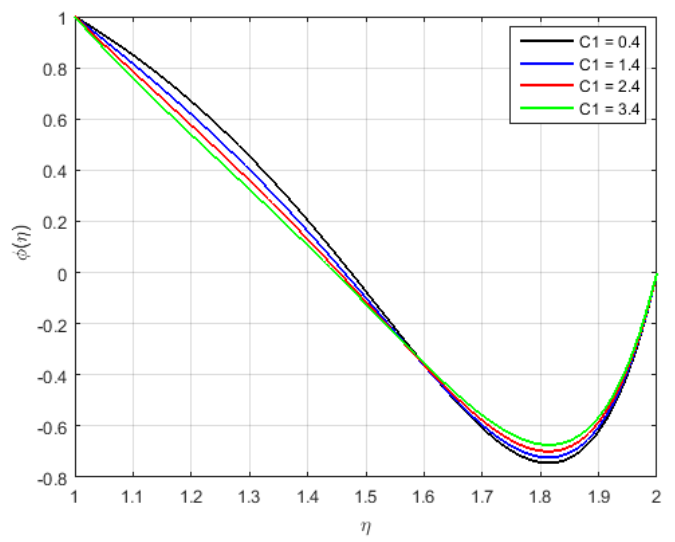

Figure 11: Influence of $\phi(\eta)$ for $C 1$ and fixed values of $S=2.5, M=1.4, \operatorname{Pr}=2.2, \operatorname{Re} e_{m}=2.5, H s=$ $1.2, S c=1.4, S r=2.5$.

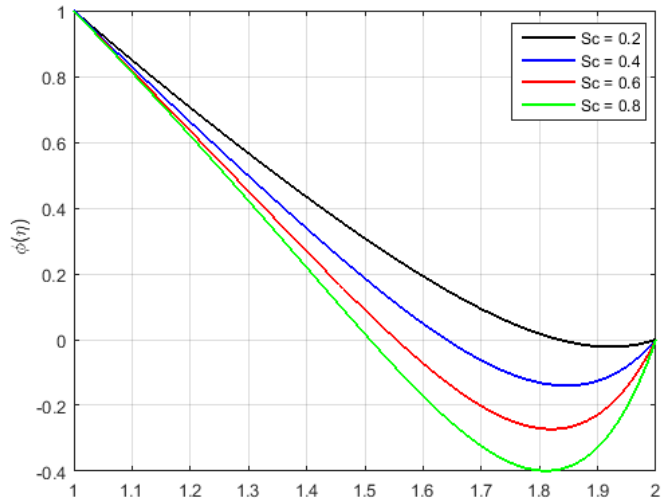

a

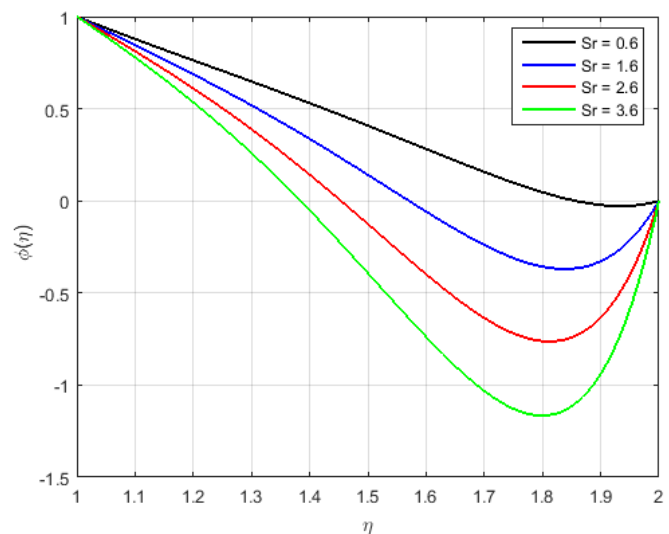

Figure 12: Influence of $\phi(\eta)$ for $S c$ and $S r$ and fixed values of $S=2.5, M=1.4, \operatorname{Pr}=2.2, \operatorname{Re}_{m}=$ $2.5, C 1=1.4, H s=1.2$.

\section{Data Availability Statement}

Not applicable.

\section{References}

[1] McCroskey, W.J., 1977. Some current research in unsteady fluid dynamics. The 1976 Freeman scholarship lecture. ASME J. Fluid Eng. 99, 8-39.

[2] Wang, C.Y., 1988. Fluid flow due to a stretching cylinder. Phys. Fluids 31, 466-468.

[3] Ishak, A., Nazar, R., Pop, I., 2008a. Uniform suction/blowing effect on flow and heat transfer due to a stretching cylinder. Appl. Math. Model. 32, 2059-2066. 

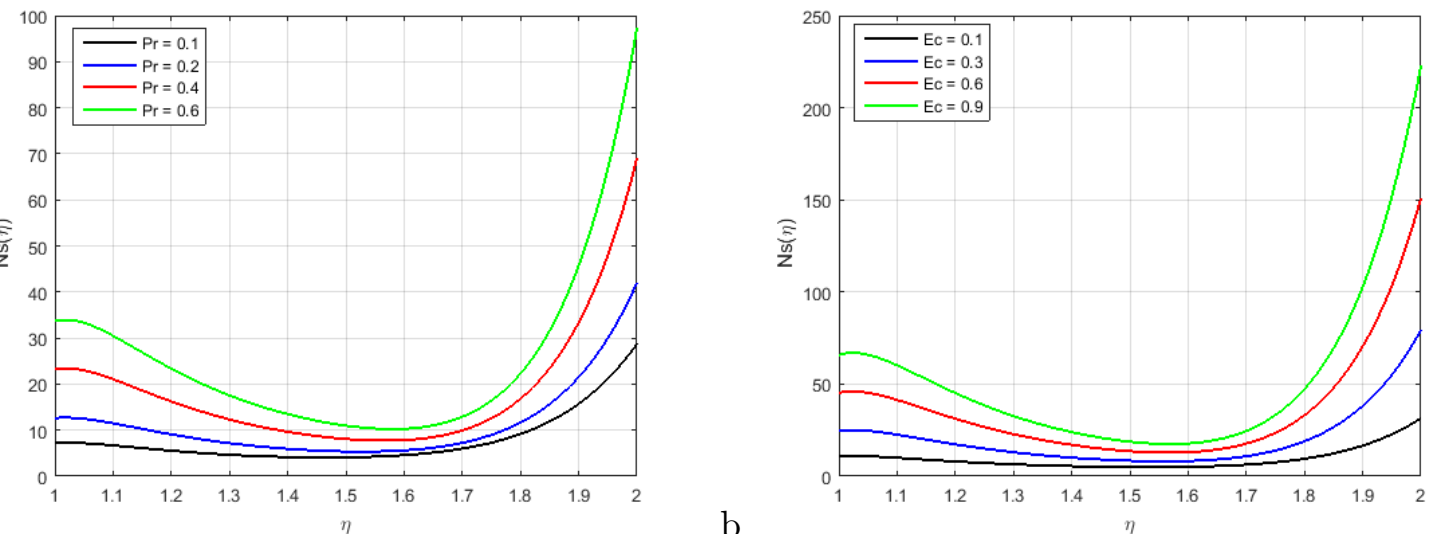

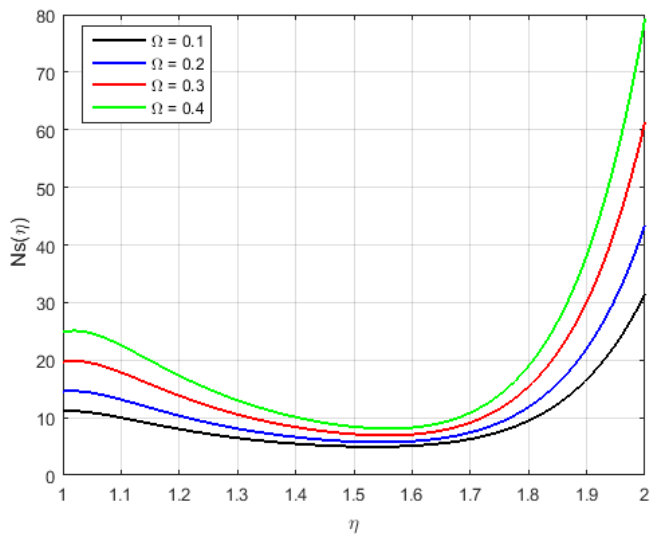

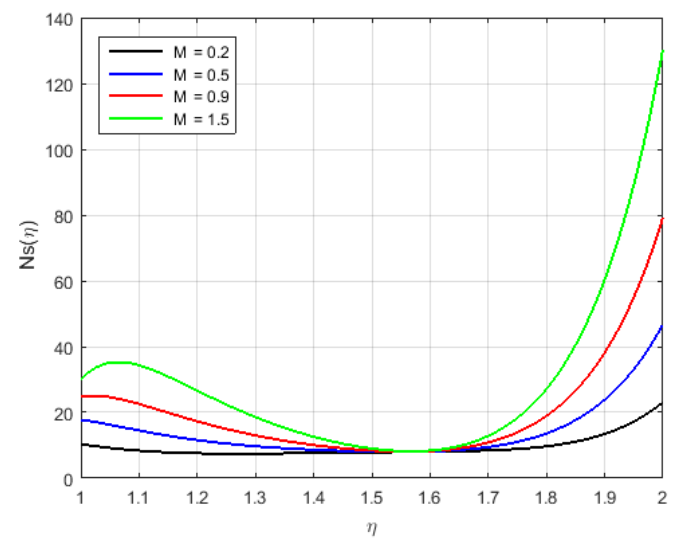

Figure 13: Influence of $N s(\eta)$ for $\operatorname{Pr}, E c, \Omega$ and $M$ and fixed values of $S=3.3, R e_{m}=1.7, C 1=$ $1.6, H s=2.2, S c=0.9, S r=1.6, \delta=0.5$.

[4] Telionis, D.P., 1981. Unsteady Viscous Flows. Springer, New York.

[5] Wang, C.Y., 1989. Exact solutions of the unsteady Navier-Stokes equations. Appl. Mech. Rev. $42,269-282$.

[6] Miklavcic, M., Wang, C.Y., 2006. Viscous flow due to a shrinking sheet. Quart. Appl. Math. 64, $283-290$.

[7] Riley, N., 1990. Unsteady viscous flows. Sci. Progress, Oxford 74, 361-377.

[8] Wang, C.Y., 2008. Stagnation flow towards a shrinking sheet. Int. J. Non-linear Mech. 43, 377-382.

[9] Fang, T., Zhang, J., Zhong, Y., Tao, H., 2011. Unsteady viscous flow over an expanding stretching cylinder. Chin. Phys. Lett. 28. Article ID 124707.

[10] Fang, T., Zhang, J., Zhong, Y., 2012. Note on unsteady viscous flow on the outside of an expanding or contracting cylinder. Commun. Nonlinear Sci. Numer. Simul. 17, 3124-3128. 

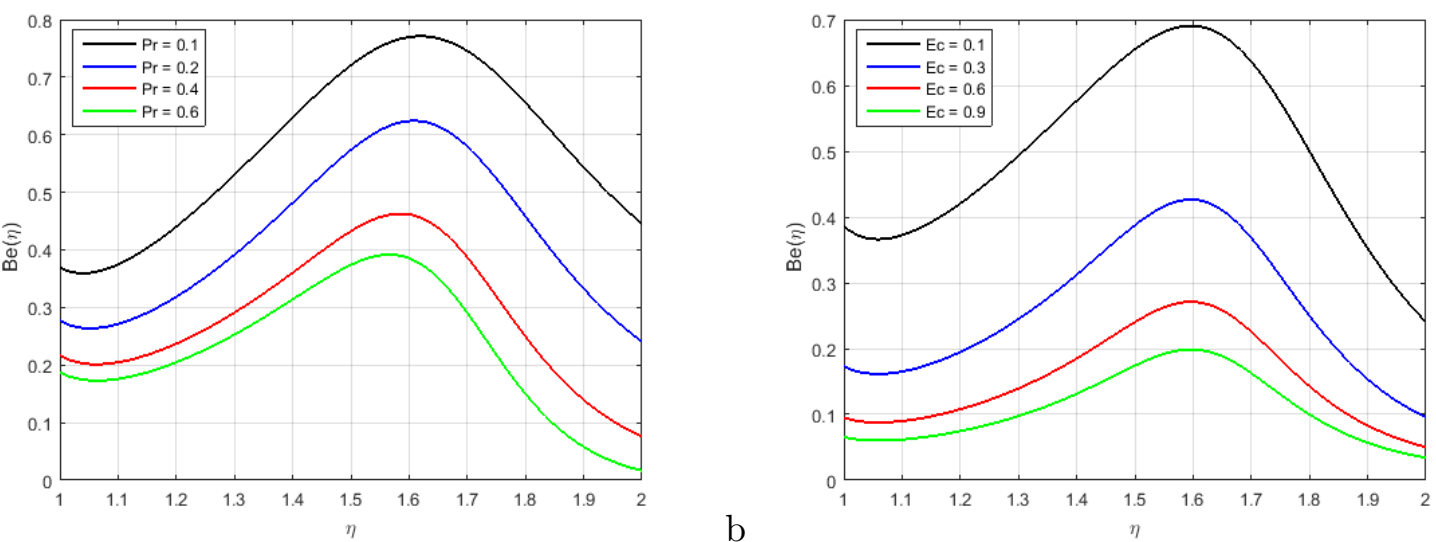

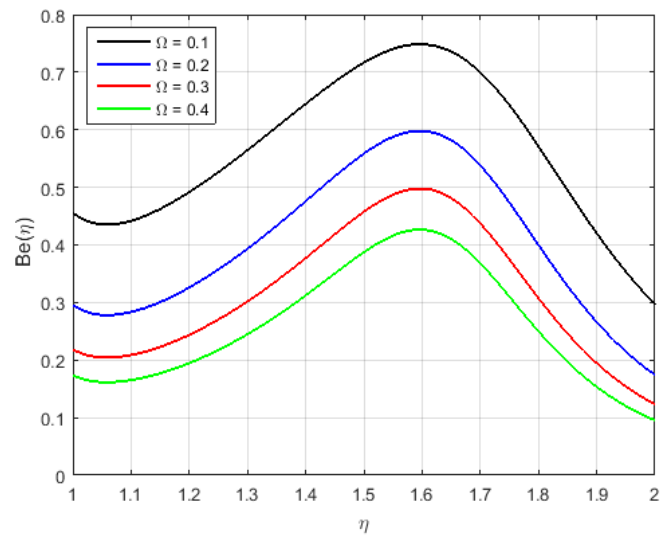

$\mathrm{b}$

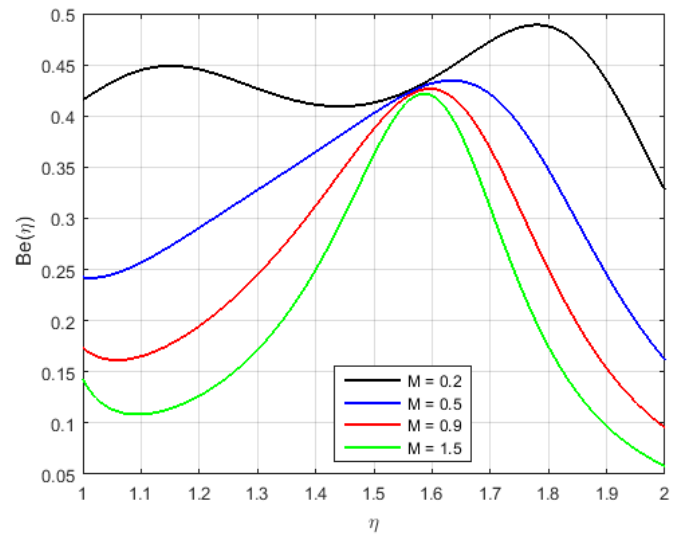

Figure 14: Influence of $B e(\eta)$ for $\operatorname{Pr}, E c, \Omega$ and $M$ and fixed values of $S=3.3, R e_{m}=1.7, C 1=$ $1.6, H s=2.2, S c=0.9, S r=1.6, \delta=0.5$.

[11] Stefan J. Versuche ber die scheinbare Adhsion. Ann Phys 1875; 230: 316-318.

[12] Domairry G and Aziz A. Approximate analysis of MHD squeeze flow between two parallel disks with suction or injection by homotopy perturbation method. Math Probl Eng 2009; 2009: 603916 .

[13] Siddiqui AM, Irum S and Ansari AR. Unsteady squeezing flow of a viscous MHD fluid between parallel plates, a solution using the homotopy perturbation method. Math Model Anal 2008; 13: $565-576$.

[14] Rashidi MM, Shahmohamadi H and Dinarvand S. Analytic approximate solutions for unsteady twodimensional and axisymmetric squeezing flows between parallel plates. Math Probl Eng 2008; 2008: 935095.

[15] Nayak AK. An analysis of steady/unsteady electroosmotic flows through charged cylindrical nano-channels. Theor Comput Fluid Dyn 2013; 27: 885-902. 
[16] Sheikholeslami M, Ganji DD and Ashorynejad HR. Investigation of squeezing unsteady nanofluid flow using ADM. Powder Technol 2013; 239: 259-265.

[17] SC Rajvanshi, BS Saini and S Wasu. Heat transfer and entropy generation on MHD squeezing flow between two parallel rotating plates using Brinkman model. J. Rajasthan Acad. Phys. Sci. $2013 ; 12,181-98$.

[18] S Sangapatnam, BR Nandanoor and RP Vallampati. Radiation and mass transfer effects on MHD free convection flow past an impulsively started isothermal vertical plate with dissipation. Therm. Sci. 2009; 13, 171-81.

[19] VR Prasad and NB Reddy. Radiation and mass transfer effects on an unsteady MHD free convection flow past a heated vertical plate in a porous medium with viscous dissipation. Theor. Appl. Mech. 2007; 34, 135-60.

[20] RAJVAnSHI, S. C., Sargam, W. A. S. U. (2014). Heat transfer in mhd squeezing flow using brinkman model. Walailak Journal of Science and Technology (WJST), 11(11), 931-938.

[21] Pattnaik PK, Mishra SR, Mahanthesh B, et al. Heat transport of nano-micropolar fluid with an exponential heat source on a convectively heated elongated plate using numerical computation. Multidiscipl Model Mater Struct 2020; 16(5): 1295-1312.

[22] Uddin MJ, Beg OA and Uddin MN. Energy conversion under conjugate conduction, magnetoconvection, diffusion and nonlinear radiation over a non-linearly stretching sheet with slip and multiple convective boundary conditions. Energy 2016; 115: 1119-1129.

[23] Hosseinzadeh Kh, Roghani So, Mogharrebil AR, et al. Optimization of hybrid nanoparticles with mixture fluid flow in an octagonal porous medium by effect of radiation and magnetic field. J Therm Anal Calorim 2021; 143: 1413-1424.

[24] Hosseinzadeh Kh, Asadi A, Mogharrebi AR, et al. Investigation of mixture fluid suspended by hybrid nanoparticles over vertical cylinder by considering shape factor effect. J Therm Anal Calorim 2021; 143: 1081-1095.

[25] Umavathi, J. C., Patil, S. L., Mahanthesh, B., Bég, O. A. (2021). Unsteady squeezing flow of a magnetized nano-lubricant between parallel disks with Robin boundary conditions. Proceedings of the Institution of Mechanical Engineers, Part N: Journal of Nanomaterials, Nanoengineering and Nanosystems, 235(3-4), 67-81. 
[26] Shah, R. A., Ullah, H., Khan, M. S., Khan, A. (2021). Parametric analysis of the heat transfer behavior of the nano-particle ionic-liquid flow between concentric cylinders. Advances in Mechanical Engineering, 13(6), 16878140211024009.

[27] Khan M. S., Rehan A. S., Aamir K., Effect of variable magnetic field on the flow between two squeezing plates. Eur. Phys. J. Plus 134: 219 (2019).

[28] Khan M. S., Rehan A. S., Amjad A., Aamir K., Parametric investigation of the Nernst-Planck model and Maxwell's equations for a viscous fluid between squeezing plates. Boundary Value Problems, 2019:107 (2019).

[29] K. Singh, K. R. Sawan, A. Kumar. Heat and Mass Transfer on Squeezing Unsteady MHD Nanofluid Flow between Parallel Plates with Slip Velocity Effect. Journal of Nanoscience, 11, (2016).

[30] A. M. Siddiqui, S. Irum, A. R. Ansari, Unsteady squeezing flow of a viscous MHD fluid between parallel plates, a solution using the Homotopy Perturbation Method. Math Model Anal, 13 (2008), 565-576.

[31] M. Sheikholeslami, M.M. Rashidi, D.D. Ganji, Numerical investigation of magnetic nanofluid forced convective heat transfer in existence of variable magnetic field using two phase model. J. Molecular Liquids, 212 (2015), 117-126.

[32] M. Sheikholeslami, M. Hatami, G. Domairry, Numerical simulation of two phase unsteady nanofluid flow and heat transfer between parallel plates in presence of time dependent magnetic field. J. Taiwan Inst. Chem. Eng., 46 (2015), 43-50.

[33] M. Sheikholeslami, D. D. Ganji, Unsteady nanofluid flow and heat transfer in presence of magnetic field considering thermal radiation. J Braz. Soc. Mech. Sci. Eng., 37, (2015), 895-902.

[34] M. O. Okongo, P. N. Gichohi, J. M. Mugambi, Hall Current Effects on a Flow in Variable Magnetic Field past an Infinite Vertical Porous Flat Plate. International Journal of Research in Applied Science, 19, (2014), 97-113.

[35] A. Bejan, Entropy Generation Through Heat Fluid Flow. New York Wiley, (1982).

[36] A. Bejan, Entropy Generation Minimization. CRC Press, New York, NY, USA, (1995).

[37] A. Hijleh, A. K. Bassam, W. N. Heilen, Entropy generation due to laminar natural convection over a heated rotating cylinder. Int. J. Heat and Mass Transfer, 42 (1999), 4225-4233. 
[38] S. H. Tasnim, S. Mahmud, M. A. H. Mamun, Entropy generation in a porous channel with hydromagnetic effect. Exergy, an Int. J., 2, (2002), 300-308.

[39] M. Q. A. Odat, A. Renhe, Damseh, A. Mohd, Al-Nimr., Effect of Magnetic Field on Entropy Generation Due to Laminar Forced Convection Past a Horizontal Flat Plate. 3, (2004), 293-303.

\begin{tabular}{|c|c|c|c|}
\hline & Nomenclature & & \\
\hline$P$ & Pressure & $E c$ & Eckert number \\
\hline$r, z$ & Cylindrical Coordinates & $\kappa$ & Thermal conductivity \\
\hline$u$ & r-axis velocity & $\mu$ & Viscosity \\
\hline$w$ & z-axis velocity & $\nu$ & Kinematic viscosity \\
\hline$t$ & time & $M$ & Magnetic parameter \\
\hline$S r$ & Soret number & $\sigma$ & Electrical conductivity \\
\hline$R e_{m}$ & Rynold's Magnetic Parameter & $\rho$ & Density \\
\hline $\operatorname{Pr}$ & Prandtl number & $H s$ & Heat Source/Sink \\
\hline$(\rho C p)$ & Specific Heat & $a(t)$ & Inner Cylinder \\
\hline$S$ & Squeezing number & $C 1$ & Chemical reaction paramter \\
\hline$b_{1}, b_{2}$ & Components of Magnetic field & $b(t)$ & Outer Cylinder \\
\hline$S c$ & Schmidt number & $\frac{T_{m}-T_{0}}{T_{h}-T_{0}}$ & Temperature difference \\
\hline
\end{tabular}

